ISSN 0103-5150

Fisioter. Mov., Curitiba, v. 25, n. 2, p. 325-331, abr./jun. 2012 Licenciado sob uma Licença Creative Commons

\title{
Efeito de quatro semanas de treinamento proprioceptivo no equilíbrio postural de idosos
}

\author{
Result of four weeks of propreoceptive training \\ in the studied postural balance of elderly
}

\section{Lilian Cristina Gomes do Nascimento ${ }^{[a]}$, Lislei Jorge Patrizzi ${ }^{[b]}$, Carla Cristina Esteves Silva Oliveira ${ }^{[c]}$}

[a] Acadêmica do curso de Fisioterapia da Universidade Federal do Triângulo Mineiro (UFTM), Uberaba, MG - Brasil, e-mail: nina.lilcg@hotmail.com

[b] Docente do curso de Fisioterapia da Universidade Federal do Triângulo Mineiro (UFTM), Uberaba, MG - Brasil, e-mail: lpatrizzi@uol.com.br

[c] Professora substituta do curso de Fisioterapia da Universidade Federal do Triângulo Mineiro (UFTM), Uberaba, MG Brasil, e-mail: carlaesteves10@hotmail.com

\section{Resumo}

Introdução: Com o aumento da idade cronológica, o corpo humano passa por um período de transformações que geram declínio de algumas capacidades físicas. Um dos principais fatores que limitam a vida do idoso hoje é a alteração no equilíbrio postural. Diante do exposto, o objetivo desta pesquisa foi analisar o efeito de quatro semanas de treinamento proprioceptivo nas medidas do equilíbrio postural de idosos a fim de determinar a efetividade de uma estratégia simples de intervenção. Materiais e métodos: Participaram deste estudo nove idosos, de ambos os gêneros, cadastrados na Unidade de Atenção ao Idoso da cidade de Uberaba, MG, Brasil, com média de idade de 72,87 $( \pm 5,38)$ anos, os quais foram submetidos a um treinamento proprioceptivo que consistiu em treino de marcha em pista com aproximadamente oito metros, composta por quatro tipos de solo com dificuldades progressivas. A frequência de treinamento foi de duas vezes por semana, com duração de 20 minutos por sessão, durante quatro semanas. Resultados: Após quatro semanas de treinamento, houve diminuição significativa nas oscilações durante a realização do Romberg com olhos abertos em solo estável $(p=0,001)$, com olhos fechados em solo estável $(p=0,02)$ e com olhos fechados em solo instável $(p=0,002)$; evolução no escore da escala de equilíbrio de Berg $(p=0,001)$ e um aumento na velocidade da marcha na pista $(p=0,00001)$. Conclusão: A partir dos 
resultados encontrados, concluiu-se que o treinamento proprioceptivo proposto foi eficaz para o equilíbrio postural de idosos.

Palavras-chave: Idoso. Equilíbrio postural. Propriocepção.

\section{Abstract}

Introduction: With the increase of chronological age, the human body goes through a period of transformations that generate decline of some physical capacities. One of the factors that limit the life of elderly nowadays is the postural balance. Thus, the objective of this research was to analyze the effect of four weeks of a proprioceptive training program in the measures of the postural balance of aged people, in order to determine the effectiveness of a simple strategy of intervention. Materials and methods: Nine aged people of both genders registered in the unit of attention to the third aged in the city of Uberaba, MG - Brazil, with the average age of $72,8( \pm 5,38)$ years, who were submitted to a proprioceptive training that consisted approximately of trainings through a march in a track with eight meters, approximately, composed of four types of ground with gradual difficulties. The training frequency was twice a week, 20 minutes each session, during four weeks. Results: After four weeks of training, there was significant decrease in the variation during the Romberg's application with eyes closed in stable ground ( $p=0.001)$, with eyes closed on stable ground ( $p=0.02)$ and with eyes closed in unstable ground ( $p=0.002)$; evolution of the score in Berg's scale of balance ( $p=0.001)$ and an increase in the speed of the march in the track $(p=0.00001)$. Conclusion: It is concluded that the proprioceptive training proposed was efficient for the postural balance of aged ones.

Keywords: Elderly. Postural balance. Proprioception.

\section{Introdução}

Um acelerado ritmo do crescimento da população idosa é observado mundialmente, inclusive no Brasil e em outros países latino-americanos $(1,2)$. Diante do envelhecimento da população, a abordagem de temas relativos à terceira idade torna-se cada vez mais relevantes.

Com o aumento da idade cronológica, o corpo humano passa por um período de transformações que geram declínio de algumas capacidades físicas, tais como a diminuição da flexibilidade, agilidade, coordenação, mobilidade articular e, principalmente, o equilíbrio $(3,4)$. 0 equilíbrio é a capacidade de manter a posição do corpo sobre sua base de apoio, seja ela estacionária ou móvel. Denomina-se equilíbrio estático o controle da oscilação postural na posição imóvel (5) e equilíbrio dinâmico o movimento do corpo de uma maneira controlada (6).

A alteração do equilíbrio favorece as quedas; sendo assim, essa alteração é considerada um dos principais fatores que limitam, hoje, a vida do idoso (7). Sabe-se que os idosos que sofrem quedas apresentam capacidade inferior para manutenção do controle postural; no entanto, mesmo os idosos que nunca sofreram quedas frequentemente admitem ter dificuldade em manter-se equilibrados e estáveis (8). Em $80 \%$ dos casos de quedas, esse fato não pode ser atribuído a uma causa específica, mas a um comprometimento do sistema de equilíbrio como um todo (9).

Para que a manutenção do equilíbrio ocorra, os sistemas sensoriais devem agir de forma a conduzir informações específicas, relacionadas ao posicionamento do corpo no espaço, cabendo ao sistema nervoso central (SNC) organizá-las e controlar a postura corporal tanto estática quanto dinâmica (10). Ao receber as informações sensoriais, o SNC as processa no contexto das respostas previamente aprendidas e executa uma resposta de correção postural automática, que é orientada ou expressa por meio da resposta mecânica que se apoia. A lentificação geral do processamento das informações sensoriais, associada à diminuição da condução nervosa, comuns no processo de envelhecimento, contribuem para o retardo das respostas posturais automáticas (11).

A propriocepção e a informação sensorial são fatores importantes para a manutenção do equilíbrio postural em condições normais e o treinamento proprioceptivo aumenta esses estímulos, permitindo melhor equilíbrio postural (12). Com a perda de 
propriocepção durante o envelhecimento, aumenta-se o limiar para a detecção do movimento articular, ou seja, a propriocepção.

Figueiredo et al. (13) confirmam um maior nível de comprometimento de equilíbrio funcional no grupo de idosos quando comparado ao de adolescentes, jovens e adultos. Estudos comprovam que indivíduos participantes de atividades multissensoriais com enfoque na estimulação proprioceptiva demonstraram maior estabilidade postural quando comparados a um grupo controle $(14,15)$; porém, faz-se necessário que mais estudos sejam realizados a fim de estimular a utilização de protocolos no cotidiano dos fisioterapeutas com objetivos práticos, como prescrição de exercícios em toda a população idosa (16).

O objetivo desta pesquisa foi analisar o efeito de quatro semanas de treinamento proprioceptivo nas medidas do equilíbrio postural de idosos, com o intuito de determinar a efetividade de uma estratégia simples de intervenção, promovendo a saúde por meio de independência funcional e uma visão preventiva das quedas, com melhora do equilíbrio estático e dinâmico.

\section{Materiais e métodos}

Sujeitos

Esta pesquisa caracterizou-se como estudo de intervenção, prospectivo longitudinal. Participaram do estudo nove idosos de ambos os gêneros, sendo oito mulheres e um homem, cadastrados na Unidade de Atenção ao Idoso da cidade de Uberaba, MG, Brasil, com uma média de idade de $72,87( \pm 5,38)$ anos, com independência funcional e classificados como irregularmente ativos segundo o Questionário Internacional de Atividade Física (IPAQ).

Foram estabelecidos como critérios de exclusão: doenças neurológicas, autonomia cognitiva comprometida ou capacidade de autodeterminação limitada, lesão ortopédica limitante, uso de órteses para membro inferior, deformidades nos membros inferiores, utilização de algum medicamento com ação no equilíbrio, doenças do sistema cardiorrespiratório que impossibilitassem as atividades propostas, ser portador de qualquer tipo de comprometimento à sua permanência na posição ortostática e os participantes que apresentassem duas faltas consecutivas ou um total de três ou mais faltas durante o tempo proposto para o treinamento estabelecido.
Este estudo foi aprovado pelo Comitê de Ética e Pesquisa da Universidade Federal do Triângulo Mineiro - UFTM (protocolo n. 1.429). Todos os participantes receberam as informações e orientações necessárias quanto às atividades que seriam realizadas, no que diz respeito ao objetivo da pesquisa e aos procedimentos que seriam submetidos. Após esclarecimentos, todos assinaram o termo de consentimento livre e esclarecido.

\section{Instrumentação}

Os participantes receberam orientações específicas quanto aos testes, sendo as dúvidas esclarecidas antes da realização dos mesmos. Foi solicitado aos participantes informarem ao examinador qualquer tipo de dor ou desconforto durante sua aplicação.

O equilíbrio foi avaliado no início da intervenção e após quatro semanas de treinamento, por meio dos instrumentos:

- Balance Scale ou escala de equilíbrio de Berg (EEB): escala composta por 14 itens com atividades comuns da vida diária, com grau de dificuldade progressivo relacionadas à força, flexibilidade e equilíbrio dinâmico e estático. Cada item nesta escala é graduado de 0 a 4 pontos, sendo o escore total de 56 pontos. Os pontos devem ser subtraídos caso o tempo ou a distância não sejam atingidos, o sujeito necessite de supervisão para a execução da tarefa, ou se o sujeito apoia-se num suporte externo ou recebe ajuda do examinador. Assim, uma melhora do indivíduo é caracterizada por um aumento no escore total (17);

- Romberg modificado: teste de equilíbrio estático realizado em solo estável e instável, no qual o sujeito permanece em pé por 30 segundos (18). Estão representadas na Figura 1 algumas possíveis variações do teste em que são utilizadas três posições distintas para base de apoio dos pés (19), sendo estas realizadas primeiramente de olhos abertos e depois de olhos fechados, nas seguintes condições sensoriais:

1) Romberg com olhos abertos, apoio bipodal nas distintas posições de base de apoio em superfície estável (ROA-E); 
2) Romberg com olhos abertos, apoio bipodal nas distintas posições de base de apoio em superfície instável (ROA-I);

3) Romberg com olhos fechados, apoio bipodal nas distintas posições de base de apoio em superfície estável (ROF-E);

4) Romberg com olhos fechados, apoio bipodal nas distintas posições de base de apoio em superfície instável (ROF-I).

Os sujeitos que apresentam pequenas oscilações sem risco de queda (Romberg ausente) têm uma condição normal de equilíbrio e os que apresentam a possibilidade de queda ou queda propriamente dita (Romberg presente) revelam um deficit no equilíbrio (15).

Para a análise das respostas, adotou-se que para cada condição sensorial analisada, seria dada uma pontuação inicial de 30 pontos e, a partir deste valor, seriam descontados 10 pontos para cada Romberg presente. Assim, a melhora do equilíbrio foi observada com o aumento da pontuação atribuída a Romberg decorrente da diminuição de oscilações que apresentassem risco eminente de queda.

\section{Procedimento}

O programa de intervenção consistiu em um treinamento proprioceptivo no qual os participantes realizaram a marcha individualmente, fazendo uso de tênis, em uma pista de treinamento nas dependências da Unidade de Atenção ao Idoso.

A pista de treinamento (Figura 2) foi composta por quatro tipos de solo com dificuldades progressivas e com uma distância aproximada de dois metros cada

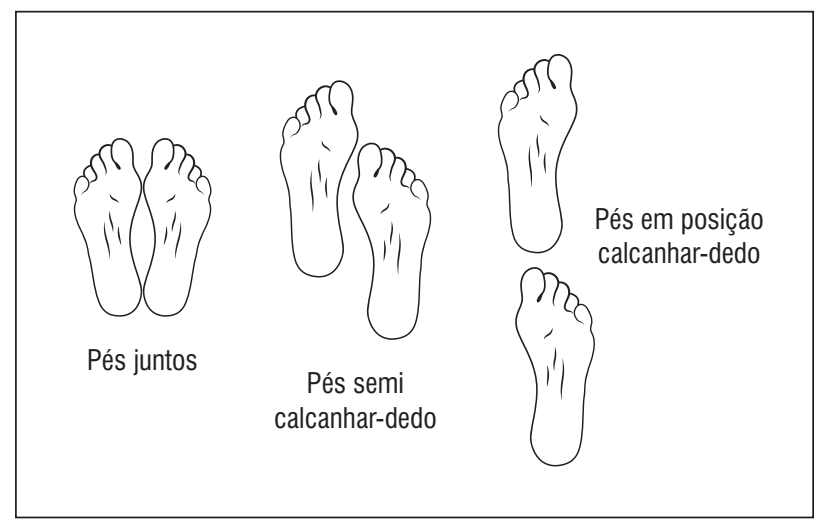

Figura 1 - Posições distintas dos pés analisadas em Romberg solo, totalizando oito metros. A frequência do treinamento foi de duas vezes por semana com duração de 20 minutos cada, durante quatro semanas, num total de oito sessões.

Como caráter de aprendizado, o participante realizava uma volta completa (do início ao final da pista, e do retorno ao início), sendo o tempo da volta seguinte cronometrado como parâmetro para análise da mudança da velocidade da marcha. Foi comparado o tempo em segundos da segunda volta da primeira sessão com a segunda volta da última sessão da intervenção.

\section{Análise estatística}

Para a análise estatística, foram utilizados os testes Anderson Darling e teste T-pareado, sendo estabelecido nível de significância de $\mathrm{p}<0,05$.

\section{Resultados}

A análise dos dados revelou grau de homogeneidade do grupo em relação à idade, peso, estatura e Índice de Massa Corporal (IMC). Os dados apresentados na Tabela 1 descrevem o perfil dos indivíduos estudados e o respectivo grau de homogeneidade.

Os resultados serão apresentados em forma de tabela (Tabela 2), a partir da comparação dos níveis de equilíbrio do grupo antes do início da intervenção (pré) e após quatro semanas de treinamento (pós).

Os resultados mostram diminuição das oscilações que representam risco de queda em Romberg $(p<0,05)$, aumento no escore total de EEB $(p=0,001)$ e menor tempo $(p=0,00001)$ para percorrer o trajeto após treinamento proprioceptivo.

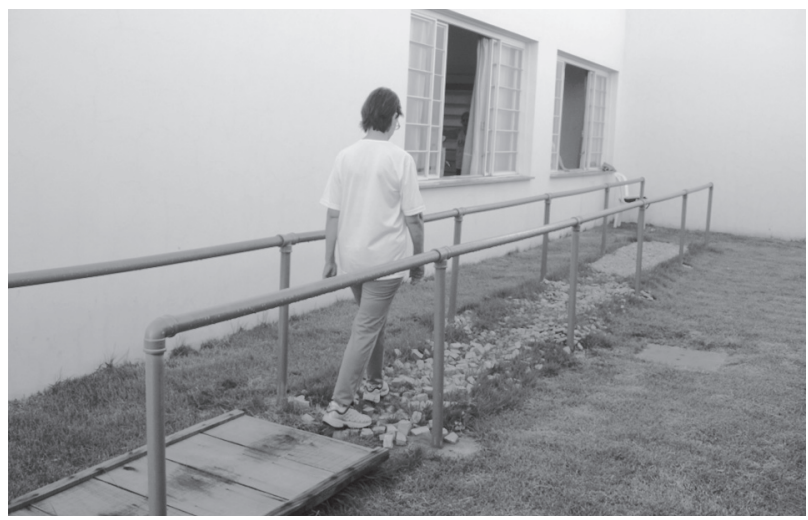

Figura 2 - Idosa durante marcha na pista de treinamento 


\section{Discussão}

Muitos estudos têm sido direcionados à efetividade de intervenções sobre o treino de equilíbrio em idosos, uma vez que os deficits de equilíbrio constituem um fator de risco que pode ser modificável por meio de uma intervenção baseada em exercícios (20). Participaram deste estudo 15 idosos, porém, os dados de quatro participantes foram excluídos por apresentarem duas faltas consecutivas, e dois foram excluídos por apresentarem três faltas no decorrer do período de treinamento, sendo que nove permaneceram até o final do trabalho.

A reabilitação do equilíbrio é baseada na exposição do idoso repetidamente às condições de dificuldades (21).

Os principais resultados obtidos neste estudo demonstram uma diminuição das oscilações que representassem risco de queda aos idosos em ROA-E $(\mathrm{p}=0,001)$, ROF-E $(\mathrm{p}=0,02)$ e ROF-I $(\mathrm{p}=0,002)$ comprovando que idosos expostos a condições de dificuldades melhoram o seu equilíbrio estático. Há

Tabela 1 - Análise descritiva da amostra: idade (anos), peso $(\mathrm{kg})$, estatura $(\mathrm{cm})$ e Índice de Massa Corpórea (IMC)

\begin{tabular}{lcrc}
\hline Características & Média & $\begin{array}{r}\text { Desvio } \\
\text { padrão }\end{array}$ & Homogeneidade \\
\hline Idade & 72,87 & 5,38 & 0,105 \\
Peso & 66,4 & 16,09 & 0,246 \\
Estatura & 159,83 & 4,35 & 0,130 \\
IMC & 68,5 & 16,57 & 0,184 \\
\hline
\end{tabular}

Fonte: Dados da pesquisa. evidências de que exercícios direcionados para a estimulação do equilíbrio mostraram-se eficazes na recuperação e manutenção do equilíbrio no processo de envelhecimento (22).

Após a intervenção, os participantes deste estudo apresentaram um aumento no escore total de EEB, de $50,8( \pm 2,4)$ para $53,6( \pm 1,5)$, demonstrando significante melhora na condição do equilíbrio dinâmico e estático, levando a uma diminuição na possibilidade de queda.

Santos et al. (23) relatam que quanto menor o desempenho na EEB, maior é a probabilidade de queda em idosos, e confirmam que um idoso com uma pontuação inferior a 49 tem aproximadamente dez vezes mais chances de sofrer queda do que aqueles com pontuação superior. No entanto, idosos que realizam um programa de treinamento motor apresentam melhora no equilíbrio, o que possivelmente diminui o risco de quedas e aumenta a independência nas atividades diárias (24). Zambaldi et al. (20) observaram melhora das medidas de equilíbrio e nos resultados da EEB após treinamento de equilíbrio, de curto período, com tarefas específicas e sem associações de treino de força muscular, o que acarretaria também uma prevenção do risco de quedas.

A redução da velocidade de marcha em idosos tem sido associada a uma diminuição da capacidade de controlar o equilíbrio corporal (21). Um estudo de coorte avaliou a velocidade de marcha de um grupo de idosos, distinguindo-os em três níveis de velocidade (alta, média e baixa). Autores verificaram que o grupo de menor velocidade de marcha apresentou alta incidência de quedas entre outros eventos adversos $(25,26)$. Gauchard et al. (27) demonstraram que uma intervenção de exercícios com ênfase na propriocepção em mulheres com média de idade de 60 anos,

Tabela 2 - Média, desvio padrão pré e pós-intervenção e p-valor dos testes ROA-E (Romberg com olhos abertos em solo estável), ROA-I (Romberg com olhos abertos em solo instável), ROF-E (Romberg com olhos fechados em solo instável), ROF-I (Romberg com olhos fechados em solo instável), EEB (Escala de equilíbrio de Berg) e TEMPO (em segundos, referente a uma volta completa na pista)

\begin{tabular}{lcccccc}
\hline & ROA-E & ROA-I & ROF-E & ROF-I & EEB & Tempo \\
\hline Pré & 20 & $16,6( \pm 5)$ & $14,4( \pm 5,2)$ & $10( \pm 7)$ & $50,8( \pm 2,4)$ & $45,7( \pm 10,6)$ \\
Pós & $27,7( \pm 4,4)$ & $22,2( \pm 4,4)$ & $21,1( \pm 6)$ & $18,8( \pm 6)$ & $53,6( \pm 1,5)$ & $36,1( \pm 8,2)$ \\
p-valor & $0,001^{*}$ & 0,05 & $0,02^{*}$ & $0,002^{*}$ & $0,001^{*}$ & $0,00001^{*}$ \\
\hline
\end{tabular}

Fonte: Dados da pesquisa.

Legenda: * = Diferença significativa $(p<0,05)$. 
traz melhoras significantes nos padrões de equilíbrio postural em relação a exercícios de somente de caminhada e corrida. Lanuez e Filho (28) relatam que a melhora do equilíbrio em seu grupo praticante de exercícios aeróbios deve-se ao fato das caminhadas terem sido realizadas em terrenos abertos e irregulares, ocorrendo, assim, estímulos em diversos sistemas responsáveis pelo controle postural.

Os dados acima descritos confirmam os resultados desta pesquisa, na qual a diminuição do tempo gasto pelos idosos para percorrer a pista, de 45,7 $( \pm 10,6)$ para $36,1( \pm 8,2)$ segundos, foi decorrente do aumento da velocidade da marcha dos participantes, sugerindo que o mesmo pode ter sido ocasionado pela melhora do equilíbrio dos mesmos em função dos estímulos proprioceptivos do treinamento.

Melhorando a função dos idosos, é provável que haja diminuição do número de quedas (18). Santos et al. (29) afirmam que a melhor forma de intervir sobre a queda seria realizando sua prevenção por meio de exercícios de fácil aplicação, baixo custo, de caráter preventivo e curativo em relação às alterações do equilíbrio.

Estes achados evidenciam a importância da inserção de treinamentos que busquem a melhora do equilíbrio da população idosa, uma vez que tal grupo pode ser beneficiado pelo programa de treinamento proprioceptivo, minimizando os efeitos fisiológicos do envelhecimento. No entanto, conforme afirmam Brandalize et al. (30), ao se interromper o programa de exercícios, os ganhos são perdidos. Com isso, é muito importante que, com o planejamento dos exercícios, se fizesse um planejamento para a aderência dos idosos aos programas.

Sugerimos que novas pesquisas que confirmem a melhora do equilíbrio e a diminuição de possibilidade de quedas em idosos sejam realizadas e que estas envolvam uma amostragem mais significativa, façam uso de maior tempo de intervenção e utilizem indivíduos classificados nos variados níveis de atividade física para apoiar ou refutar seus achados. Os fatos descritos podem justificar a diferença não significante obtida no ROA-I ( $\mathrm{p}=0,05)$.

Uma limitação deste estudo que deve ser considerada é o fato de se utilizarem apenas testes de campo para avaliar as variáveis estudadas, não podendo contar com mensurações mais objetivas realizadas em testes de laboratório. No entanto, isso pode ser em razão de a intervenção não ter sido duradoura o suficiente para que se obtivesse melhora nesse item ou, ainda, pode ser decorrente do pequeno número de participantes.

\section{Conclusão}

A partir dos resultados encontrados neste estudo, pode-se concluir que a eficácia do treinamento proprioceptivo específico acarreta melhora significante no escore total de EEB, diminuição de oscilações que representem riscos de queda em Romberg, diminuição do tempo para percorrer determinada distância e, consequentemente, melhora no equilíbrio estático e dinâmico de idosos irregularmente ativos.

Diante do exposto, pode-se supor que o treinamento proprioceptivo apresenta uma oportunidade para prevenção de quedas e promoção à saúde por meio de independência funcional da população idosa.

\section{Referências}

1. Ramos LR, Rosa TEC, Oliveira ZM, Medina MCG, Santos FRG. Perfil do idoso em área metropolitana na região sudeste do Brasil: resultados de inquérito domiciliar. Rev Saúde Pub. 1993;27:87-94.

2. Pacheco R, Santos SSC. Avaliação global de idosos em unidades de PSF. Textos sobre Envelhecimento 2004;7(2):9-31.

3. Mann L, Kleinpaul J, Teixeira CS, Rossi AG, Lopes LFD, Mota CB. Investigação do equilíbrio corporal em idosos. Rev Bras Geriatr Gerontol. 2008;11(2):1809-23.

4. Matsudo SM, Matsudo VKR. Prescrição e benefícios da atividade física na terceira idade. Bras Ciên Mov. 1992;6(4):19-30.

5. Cyarto EV, Brown WJ, Marshall AL, Trost SG. Comparison of the effects of a home-based and group-based resistance training program on functional ability in older adults. Am J Health Promot. 2008;23(1):13-7.

6. Barela JA. Estratégias de controle em movimentos complexos: ciclo percepção-ação no controle postural. Rev Paul Educ Fís. 2000;(Supl. 3):79-88.

7. Bittar RSM, Pedallini MEB, Bottino MA, Formigoni LG. Síndrome do desequilíbrio no idoso. Pró-Fono. 2002; 14(1):119-28.

8. Barrett-Connor E, Weiss TW, McHorney CA, Miller PD, Siris ES. Predictors of falls among postmenopausal women: results from the National Osteoporosis Risk Assessment (NORA). Osteoporos Int. 2009;20(5): 715-22. 
9. Alexander NB. Postural control in older adults. J Am Geriatr Soc. 1994;42(1):93-108.

10. Mccollum C, Shupert CL, Nashner LM. Organizing sensory information for postural control in altered sensory environments. Journal of Biology. 1996;180(3):257-70.

11. Chandler JM. Equilíbrio e quedas no idoso: questões sobre a avaliação e o tratamento. In: Guccione AA. Fisioterapia geriátrica. Rio de Janeiro: Guanabara Koogan; 2002. p. 265-77.

12. Carvalho J, Pinto J, Mota, J. Atividade física, equilíbrio e medo de cair. Um estudo em idosos institucionalizados. Rev Port Cien Desp. 2006;7(2):225-31.

13. Figueiredo LL, Pícoli TS, Borges APO, Patrizzi LJ. Análise do equilíbrio no processo de envelhecimento. Fisioter Mov. 2011;24(3):401-7.

14. Hu MH, Woollcott MH. Multisensory training of standing balance in older adults: postural stability and oneleg stance balance. J Gerontol. 1994;49(2):M52-M61.

15. Rogers ME, Fernandez JE, Bohlken, RM. Training to reduce postural sway and increase functional reach in the elderly. J Occup Rehab. 2001;11(4):291-8.

16. Andrade CH, Santos JCV, Corso SD. Avaliação do equilíbrio funcional de idosas institucionalizadas por meio da escala de Berg e do test timed up and go. Ter Man. 2010;8(36):121-5.

17. Berg KO, Wood-Dauphinee SL, Williams JI, Gayton D. Measuring balance in the elderly: validation of an instrument. Can J Public Health. 1992;83(2):S7-S11.

18. Tinetti ME, Baker DI, McAvay G, Claus EB, Garrett P, Gottschalk $\mathrm{M}$, et al. A multifactorial intervention to reduce the risk of falling among elderly people living in the community. N Engl J Med. 1994;331(13):821-7.

19. Ramos BMB. Influência de um programa de atividade física no controle do equilíbrio de idosos [monograph]. São Paulo: Monografia, 2005.

20. Zambaldi PA, Costa TABN, Diniz GCLM, Scalzo PL. Efeito de um treinamento de equilíbrio em um grupo de mulheres idosas da comunidade: estudo piloto de uma abordagem específica, não sistematizada e breve. Acta Fisiatr. 2007;14(1):17-24.

21. Abreu SSE, Caldas CP. Velocidade de marcha, equilíbrio e idade: um estudo correlacional entre idosas praticantes e idosas não praticantes de um programa de exercícios terapêuticos. Rev Bras Fisioter. 2008;12(4):324-30.
22. Souza ACS, Paiva PB, Guth VJ, Martins AC, Santos GM, Mazo GZ. 0 efeito do treinamento sensório-motor no equilíbrio de idosas. Rev Digital Buenos Aires. 2009 [acesso 20 nov. 2011];14(134). Disponível em: http:// www.efdeportes.com/efd134/treinamento-sensorio-motor-no-equilibrio-de-idosas.htm.

23. Santos GM, Souza ACS, Virtuoso JF, Tavares GMS, Mazo GZ. Escala de equilíbrio de Berg no risco de queda em idosos. Rev Bras Fisioter. 2011;15(2):95-101.

24. Soares MA, Sacchelli T. Efeitos da cinesioterapia no equilíbrio de idosos. Rev Neurocienc. 2008;16(2): 97-100.

25. Wolf B, Feys H, De Weerdt, van der Meer J, Noom M, Aufdemkampe G, et al. Effect of a physical therapeutic intervention for balance problems in the elderly: a single-blind, randomized, controlled multicentre trial. Clin Rehabil. 2001;15(6):624-36.

26. Montero-Odasso M, Schapira M, Soriano ER, Varela M, Kaplan R, Camera LA, et al. Gait velocity as a single predictor of adverse events in healthy seniors aged 75 years and older. J Gerontology A Biol Sci Med Sci. 2005;60(10):1304-9.

27. Gauchard GC, Gangloff P, Jeandel C, Perrin PP. Influence of regular proprioceptive and bioenergetic physical activities on balance control in elderly women. J Gerontol A Biol Sci Med Sci. 2003;58(9):M846-50.

28. Lanuez FV, Filho WJ. Efeitos de dois programas de exercícios físicos nos determinantes de aptidão motora em idosos sedentários. Einstein. 2008; 6(1):76-81.

29. Santos AC, Ferreira CP, Silva KC, Araújo VV, Lima F. Exercícios de Cawthorne e Cooksey em idosas: melhora do equilíbrio. Fisioter Mov. 2008;21(4):129-36.

30. Brandalize D, de Almeida PHF, Machado J, Endrigo R, Chodur A, Israel VL. Efeitos de diferentes programas de exercícios físicos na marcha de idosos saudáveis: uma revisão. Fisioter Mov. 2011;24(3):549-56.

Recebido: 20/04/2011 Received: 04/20/2011

Aprovado: 25/10/2011 Approved: 10/25/2011 